\title{
Disminuir la pérdida de alimentos en la producción agrícola mediante la deshidratación
}

\section{Reduce the loss of food in the agricultural production through dehydration}

\author{
Mónica Leticia Acosta Miranda 1 https://orcid.org/0000-0001-5564-8523, \\ Leonor Ángeles Hernández ${ }^{1}$ https://orcid.org/0000-0002-7316-3906 \\ ${ }^{1}$ Tecnológico Nacional de México / Instituto Tecnológico de Cuautla, Morelos, \\ México \\ monica.acosta@cuautla.tecnm.mx, \\ leonor.angeles@cuautla.tecnm.mx
}

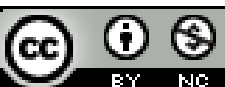

Esta obra está bajo una licencia internacional Creative Commons Atribución-NoComercial 4.0. $\begin{array}{ll}\text { Enviado: } & 2021 / 11 / 05 \\ \text { Aceptado: } & 2021 / 12 / 27 \\ \text { Publicado: } & 2021 / 12 / 30\end{array}$

\section{Resumen}

Con la intención de contribuir al logro de los Objetivos de Desarrollo Sostenible (ODS) de la agenda 2030, principalmente el ODS 12 "Producción y consumo responsables", se trabaja en el diseño y construcción de un deshidratador solar automatizado programable, que permita el aprovechamiento de los desechos generados por aquellos productos agrícolas que no llegan a los centros de consumo, por no cumplir con los estándares de venta o porque los precios del mercado se encuentran por debajo de los costos que implica la cosecha y, finalmente, terminan convertidos en basura. El prototipo de deshidratador funciona con energía solar y cuenta con sensores que le permiten seguir el movimiento del sol. Es posible programarlo para controlar los niveles de temperatura y humedad de los productos, sin requerir de personal que vigile constantemente su funcionamiento, todo esto mediante la implementación del Internet de las cosas. Puede escalarse para diferentes niveles de producción, adecuándose a los espacios de que dispongan los productores. Se realizaron pruebas con jitomate rebanado, logrando deshidratar siete kilos en 30 horas a una temperatura ambiente de 26 grados centígrados. Se pretende llevar el prototipo a diversos productores agrícolas del Oriente del Estado de Morelos para el aprovechamiento de sus excedentes lo que a su vez les permitirá ofrecer al consumidor final un producto orgánico de calidad.

Palabras clave: Deshidratador solar, Objetivos de Desarrollo Sostenible, Pérdida de alimentos, producción agrícola.

Sumario: Introducción, Materiales y Métodos, Resultados y Discusión y Conclusiones.

Como citar: Acosta, M. \& Hernández, L. (2021). Disminuir la pérdida de alimentos en la producción agrícola mediante la deshidratación. Revista Tecnológica - Espol, 33(3), 139-151.

http://www.rte.espol.edu.ec/index.php/tecnologica/article/view/874 


\begin{abstract}
This work contributes to the achievement of the Sustainable Development Goals (SDGs) of the 2030 agenda, the SDG 12 "Responsible Consumption and Production". It is the design and construction of a programmable automated solar dehydrator that allows using the waste generated by agricultural products that do not reach the centres of consumption either because they do not meet the sales standards or because market prices are below the harvest costs. Those wasted products end up becoming garbage. The prototype dehydrator is solar-powered and has sensors that allow following the movement of the sun. It can be programmed through the implementation of the Internet of Things to control the temperature and humidity levels of the products, without requiring personnel to constantly monitor its operation. It can be scaled for different production levels according to the producers' space availability. Tests were carried out with sliced tomatoes and managed to dehydrate seven kilos in 30 hours at room temperature of 26 degrees Celsius. The aim is to take the prototype to various agricultural producers in the eastern part of the state of Morelos to make use of their surpluses, which in turn will enable them to offer a quality organic product to end consumers.
\end{abstract}

Keywords: Solar dehydrator, Sustainable Development Goals, food loss, agricultural production.

\title{
Introducción
}

En la actualidad, cada vez hay menos oportunidades de trabajo para los egresados de las Instituciones de Educación Superior. La industrialización y la automatización se están apoderando de la sociedad; sin embargo, "México es el décimo segundo lugar a nivel mundial en la producción de alimentos y séptimo lugar en la exportación de alimentos" (Carreño, 2020) y la actividad agrícola en México y en el estado de Morelos se ha mantenido activa y en crecimiento pese a la Contingencia Sanitaria que ha aquejado al mundo entero desde el año 2020, apoyados en la innovación y el uso creativo del conocimiento, buscando soluciones a los problemas actuales del sector.

Uno de estos problemas es el desperdicio causado por aquellos productos que no cumplen con los estándares de color, tamaño y apariencia que el mercado exige o por los cambios en los precios de los productos en el mercado, lo que no hace rentable la recolección de la producción y, en muchas ocasiones se deja secar en el lugar de siembra hasta la siguiente temporada, momento en que se limpian los terrenos y se elimina la "basura" de la producción anterior llevándola, en la mayoría de los casos, a vertederos abiertos en los cuales se suma a las toneladas de desperdicios producidas por las actividades cotidianas contaminando el medio ambiente.

Buscando una solución a este problema se realiza el proyecto de creación de una microempresa, dedicada al diseño y fabricación de deshidratadores solares para el aprovechamiento de los excedentes de producción agrícola, que ahora son desperdicio, disminuyendo los costos y proporcionando beneficios económicos a los productores agrícolas de la región oriente del estado de Morelos aprovechando los recursos propios de la región y, generando con ello, oportunidades de autoempleo y de mayores ingresos para sus familias.

Pese a ser uno de los estados más pequeños de la República Mexicana, el estado de Morelos cuenta con un área de 135 mil hectáreas para cultivo (La redacción, 2018) una producción agrícola importante, obteniendo, en 2017, el primer lugar nacional en la producción de: Higo, nopal, caña, nochebuenas, rosas y arroz (Más de Morelos, 2017); además de un nivel importante nivel de producción de jitomate, durazno, pepino, calabaza, sorgo, elote y aguacate, entre otros; sin embargo, como se mencionó anteriormente, año con año mucha de esa 
producción es desaprovechada. Con la propuesta de utilizar el deshidratador solar la historia puede cambiar, ya que con este tipo de proyecto la fruta es deshidratada logrando una vida de anaquel más larga para su comercialización y consumo, ayudando también al medio ambiente reduciendo la cantidad de desechos y mediante un proceso que solo requiere de energía solar, abundante la mayor parte del tiempo en el Estado.

La propuesta consiste en el diseño, construcción y comercialización de un prototipo de deshidratador solar para el excedente de la producción agrícola de la Región Oriente del Estado de Morelos, con la finalidad de lograr un mayor aprovechamiento agroindustrial, evitando la pérdida de los productos, ampliando la oferta y mejorando la calidad de vida de los productores. El prototipo de deshidratador aprovecha la energía solar en el proceso, puede escalarse de acuerdo con las condiciones y requerimientos de cada productor, tiene un costo de producción accesible, está diseñado implementando el Internet de las Cosas (IoT) mediante controles programables, y puede aplicarse a diversos tipos de productos agrícolas, considerando que las frutas y verduras son el alimento más desperdiciado en el planeta, ya que "más del 15\% de la tierra cultivable en México es utilizada para producir alimentos que no será consumidos" (Pacto por la comida, s/f).

El trabajo se desarrolló en la Región Oriente del Estado de Morelos, en los municipios de Tlalnepantla, Totolapan, Atlatlahucan, Yecapixtla, Ocuituco, Jantetelco y Tetela del Volcán; municipios con una alta producción agrícola de jitomate, durazno e higo; productos que fácilmente pueden deshidratarse y, con la finalidad de apoyar al menos a un pequeño grupo de productores de esta Región que, mediante el aprovechamiento de los excedentes de producción, puedan reducir costos, diversificar sus productos y disminuir los residuos generados por el producto no cosechado al no cumplir con los estándares de comercialización.

La idea se apoyó en tres de los 17 Objetivos para el Desarrollo Sostenible (ODS) de la Agenda 2030: el objetivo 2) Hambre cero, el objetivo 11) Ciudades y comunidades sostenibles y el objetivo 12) Producción y consumo responsables. La agenda 2030, aprobada en el marco de la Cumbre de las Naciones Unidas sobre el Desarrollo Sostenible en Nueva York el 25 de septiembre de 2015, incluye los 17 Objetivos del Desarrollo Sostenible (ODS) con el objetivo general de poner fin a la pobreza, luchar contra la desigualdad y la injusticia, y hacer frente al cambio climático para el 2030 (ONU, s/f). Mediante la firma de este documento busca combatir las desigualdades y mejorar la calidad de vida de la población a través de un desarrollo sostenible. Un aspecto muy importante de la agenda 2030 es el hecho de que involucra no solo a los gobiernos, también incluye a la sociedad civil, la academia y el sector privado al considerar que el desarrollo sostenible se basa en tres aspectos relacionados entre sí: el crecimiento económico, la inclusión social y la sostenibilidad ambiental.

En la década de los años 80 , prevalecía la idea de que era necesario producir más para alimentar a la creciente población mundial; sin embargo, en los últimos años se detectaron dramáticas cantidades de desperdicio, las cuales serían suficientes para atender a todos los habitantes del planeta. Por otra parte, las frutas y verduras son el alimento más desperdiciado en el mundo. De acuerdo con la FAO (Rivera López, 2018), mil 300 millones de toneladas se desperdician anualmente, equivalente al 44 por ciento de la producción. Los tubérculos, los cereales y la leche también representan altos niveles de despilfarro.

El Objetivo de Desarrollo Sostenible (ODS) doce (12) de la Agenda 2030, refiere "garantizar condiciones de consumo y producción sostenible", sin embargo, los productores agrícolas del Estado de Morelos, como primer eslabón en la cadena de suministro, muestran pérdidas y desperdicio de productos desde la cosecha por la incertidumbre sobre los precios de 
venta que su producto puede alcanzar en el mercado, pues en ocasiones estos disminuyen a tal nivel que resulta incosteable el cosechar, abandonando la producción, que se convierte en desperdicio y se acumula a las inmensas cantidades de basura generadas por las actividades humanas cotidianas. (ODS, s/f):

Si se analizan las metas del ODS 12, el proyecto contribuye a varias de ellas como

12.2 De aquí a 2030, lograr la gestión sostenible y el uso eficiente de los recursos naturales

12.3 De aquí a 2030, reducir a la mitad el desperdicio de alimentos per capita mundial en la venta al por menor y a nivel de los consumidores y reducir las pérdidas de alimentos en las cadenas de producción y suministro, incluidas las pérdidas posteriores a la cosecha

12.5 De aquí a 2030, reducir considerablemente la generación de desechos mediante actividades de prevención, reducción, reciclado y reutilización

El Objetivo 12 "Producción y consumo responsable", pretende garantizar modalidades de consumo y producción sostenibles, debido a que el progreso económico y social de los últimos tiempos ha degradado el ambiente poniendo en peligro los sistemas de los que depende nuestro desarrollo. Cada vez son más los recursos naturales que se utilizan para apoyar las actividades económicas sin que este uso sea realmente eficiente y se genera una cantidad mayor de residuos en todo el mundo desperdiciando alrededor de un tercio de los alimentos producidos para el consumo humano, sobre todo en los países desarrollados. Se requiere mejorar la eficiencia de los recursos, minimizar los residuos e integrar las prácticas de sostenibilidad en todos los sectores de la economía reduciendo la "huella material, que es la cantidad total de materia prima extraída para satisfacer la demanda de consumo final" (Comercio justo, 2019). La huella material mundial ha aumentado el $70 \%$ desde el año 2000 y un 113\% desde el año 1990 (ONU, 2019) lo que significa un ritmo más rápido que la población y la producción económica. Es urgente reducir estas cifras y, si no es posible evitar la sobreproducción, si se pueden generar nuevas alternativas de aprovechamiento. El consumo y la producción sostenible impacta en el progreso de los otros ODS generando beneficios sociales que apoyen a una transformación.

$\mathrm{Si}$ se revisan con atención los ODS prácticamente todos se encuentran interrelacionados. En este caso, también se logra un impacto en el objetivo 11, "Ciudades y comunidades sostenibles" al reducir los desechos contaminantes y en el objetivo 2, "Hambre cero", al aprovechar esos recursos destinándolos a otros sectores de la población que actualmente se encuentran desprotegidos.

Algunas alternativas para abatir la merma de alimentos desde el lugar de la producción es la deshidratación que permite el aprovechamiento de esos productos hasta ahora desaprovechados. La deshidratación presenta ventajas prácticas y económicas ya que, al contar con un medio para conservar los alimentos, el desperdicio que se genera por la sobreproducción se ve reducido, se pueden aprovechar los productos en temporada, cuando se abaratan y conservarlos deshidratados para utilizarlos cuando estén fuera de temporada mejorando la alimentación y la economía, promoviendo el consumo de los productos de la región y ayudando a los productores en el aprovechamiento de sus cosechas. 


\section{La deshidratación}

Desde los tiempos más antiguos el hombre ha utilizado la deshidratación de alimentos como un mecanismo de conservación de alimentos, ya que de esta manera prolonga su vida útil generando productos con mayor valor agregado. Esto permite contar con frutas y verduras durante todo el año y evita la pérdida de los excedentes de producción y consumo. Asimismo, favorece la creación de microempresas familiares y las economías regionales. El proceso de deshidratado involucra la remoción de la mayor parte del agua del alimento para evitar la actividad enzimática y el desarrollo de microorganismos. La deshidratación genera estabilidad microbiológica y química, disminuye el peso y volumen, reduce el empaque, costos de almacenamiento y transporte, además permite el almacenamiento del producto a temperatura ambiente por largos períodos de tiempo (Cocimaniacos, 2017).

Prácticamente se puede deshidratar cualquier fruta u hortaliza. Por lo regular se deshidratan aquellos productos que tienen una vida útil corta o los excedentes de producción primaria. Entre las frutas y hortalizas frecuentemente deshidratadas encontramos: Ciruelas, uvas, damascos, duraznos, peras, manzanas, higos, piñas, arándanos, cerezas, bananas, kiwis, frutillas, choclos, arvejas, cebollas, tomates, zanahoria, zapallo, ajo, espinaca, puerro, pimientos, perejil, orégano, albahaca, repollo, zapallitos, entre otros (INTA, 2018).

Principales Variables en el proceso de Deshidratado (Orellana, 2017):

- La temperatura, caracteriza el estado térmico del aire al interior del deshidratador y la forma como este intercambia energía con el alimento o producto a procesar. Esta energía se transmite mediante la convección natural y forzada respectivamente. La convección, se define como la transferencia de calor por medio del movimiento de masas, esto desde una superficie hacia el aire en movimiento, conocido como ley de enfriamiento de Newton. Se puede dar de forma natural, por diferencia de temperaturas, o también de forma forzada o artificial por movimiento de aire gracias al uso de ventiladores. Cada tipo de alimento a procesar en un deshidratador presenta una serie de indicadores de temperatura que determinan un resultado óptimo.

- La humedad del aire describe la relación de mezcla entre el vapor de agua y aire seco presente en un volumen de aire determinado. El término de humedad relativa corresponde a la cantidad de vapor de agua contenido en el aire en relación con la máxima cantidad que sería capaz de contener a la misma temperatura y se expresa en porcentaje. La relación entre la temperatura del aire y la humedad contenida en él es inversamente proporcional. En el deshidratado de alimentos, al interior de una cámara de deshidratado, existen dos procesos de cambio de fase que afectan el contenido de agua en el aire:

- Evaporación: Se presenta cuando se proporciona energía a un cuerpo capaz de evaporarse si la presión de vapor de agua se encuentra por debajo del nivel de saturación. Al evaporarse el agua de un alimento, el vapor de agua pasa al aire aumentando su humedad relativa al mismo tiempo que absorbe energía de su superficie; disminuyendo la temperatura de esta.

- Condensación: La condensación aparecerá en cualquier superficie cuya temperatura está por debajo de la temperatura de punto de rocío, por lo general se da en los techos y paredes, pues estas son las primeras en enfriarse. Por lo anterior es necesario controlar de forma cuidadosa el proceso de deshidratación para evitar la pérdida de los valores nutricionales que nos aportan los alimentos, situación que se pretende atender automatizando el deshidratador. 
Como puede observarse la deshidratación es un proceso sencillo, que puede realizarse de diversas maneras y con múltiples ventajas para la conservación de los alimentos

\section{Producción de frutas y legumbres deshidratadas}

La vida útil de las frutas y legumbres en su estado natural es corta, debido a la susceptibilidad a microorganismos, reacciones enzimáticas y daños mecánicos, lo que hace necesario un mejor aprovechamiento a través de la aplicación de diversos métodos de conservación y el empleo de innovadoras técnicas de procesamiento que permiten conservar algunas características sensoriales y nutricionales de los productos por tiempos prolongados, cumpliendo con los requisitos de calidad del consumidor o cliente, en cuanto a conveniencia, inocuidad, duración, y precio (Cajamarca, Mendoza, \& Baño, 2019).

La deshidratación de un fruto fresco y natural reduce su contenido interno de agua, pero conserva todas sus virtudes, propiedades y vitaminas del producto fresco. En el mercado de Estados Unidos a los frutos deshidratados se los conoce como un alimento tipo snack que posee las siguientes características: fácil de llevar y de comer, de tamaño pequeño y que se consume entre comidas. Una de las principales características de los productos deshidratados es su alto contenido de fibra, son productos $100 \%$ naturales, libres de grasas y no requieren de refrigeración; lo que ha permitido a este sector un rápido crecimiento a nivel mundial y con precios muy competitivos. Los productos deshidratados son bien aceptados en países que tienen las cuatro estaciones ya que, debido al clima, es difícil encontrar frutas todo el año (Montesino, 2018). El consumo mundial de productos deshidratados como snacks nutritivos tenía proyecciones de 4 millones de toneladas para el 2020, debido a la tendencia mundial de consumo agroecológico y el combate a la obesidad. Entre los países compradores de productos deshidratados, los Estados Unidos encabeza la lista con el 12,9\% del total de importación ya que el consumo per-capital de frutas deshidratadas por habitante comprende en promedio 9,8 libra (PROECUADOR, 2018) le siguen, en importancia el mercado alemán $(8,8 \%)$, el ruso (5,8\%), y el Reino Unido (5,4\%) entre otros (Fede Fruta, 2018).

Hacer uso de la deshidratación como una alternativa para la conservación y mantenimiento de la calidad de los productos agrícolas permite además la integración de equipos multidisciplinarios con profesionales de ciencias como las administrativas (Sistemas de Gestión de Calidad), agrícolas (frutas) y mecánicas (deshidratadores), lo que ha permitido la innovación del campo agroindustrial respetando el medio ambiente, con un impulso económico y generador de empleo; logrando de esta manera obtener alimentos seguros, nutritivos, de alta calidad, atractivos y sanos (Cajamarca, Mendoza, \& Baño, 2019).

\section{La Producción agrícola en Morelos}

En Morelos el valor de la producción agrícola mantiene un crecimiento sostenido en los últimos años (El Sol de Cuernavaca, 2018). De acuerdo con el reporte del Servicio de Información Agroalimentaria y Pesquera (SIAP), de la Secretaría de Agricultura, Ganadería, Desarrollo Rural Pesca y Alimentación, en 2017 alcanzó el nivel más alto la historia al lograr un valor de nueve mil 745 millones de pesos, lo cual representa un incremento de dos mil 264 millones de pesos respecto al generado en 2012 y el valor de la producción agrícola fue de siete mil 326.65 millones de pesos (2000 Agro, 2018). De acuerdo con datos de INEGI (2019), la Región Oriente del Estado de Morelos se caracteriza por su alta producción de hortalizas ya sea a cielo abierto o en invernadero, siendo el jitomate o tomate rojo y el tomate verde los que se producen en mayor cantidad. En la zona norte se siembran productos como el higo (primer lugar en producción a nivel nacional) (Más de Morelos, 2017), durazno, la manzana, la ciruela, cítricos, calabaza. Por tal motivo se considera que los posibles usuarios del deshidratador 
pudieran ser los productores de algunos de estos cultivos ya que la mayoría de ellos pueden ser sometidos al proceso de deshidratación conservando los productos sus propiedades naturales.

Por otra parte, la situación de la merma de productos agrícolas es la misma para prácticamente todos los productos y en las diversas regiones del país, lo que puede disminuirse mediante la deshidratación de aquellos productos que no cumplen con las características que demanda en mercado en su estado fresco, pero si pueden utilizarse una vez deshidratados.

Por lo anterior se considera que este proyecto ofrece una alternativa de solución a las necesidades de las Pymes agrícolas que buscan agregar valor a sus productos y diversificar sus mercados mediante la disminución y aprovechamiento del desperdicio generado por una sobreproducción o abaratamiento de los productos, permitiendo conservar los alimentos para utilizarlos cuando estén fuera de temporada, mejorando la alimentación y economía de productores y de la comunidad a través de un mayor aprovechamiento de las cosechas.

\section{Materiales y Métodos}

Originalmente se trabajó de manera conjunta con la Asociación de Productores de Hortalizas Santa Bárbara S.P.R. de R.L. cuyo producto principal es el jitomate. La primera etapa del proyecto consistió en recabar información documental para conocer el estado del arte, construir el marco teórico y definir el mejor mecanismo para el diseño y construcción del prototipo, de la tarjeta de adquisición de datos, el circuito seguidor de luz y las tarjetas de circuitos electrónicos. Posteriormente se realizó el diseño en SolidWorks, así como la Medición de valores, calibración y conexión de las distintas tarjetas electrónicas con el panel solar, la batería y el motor y la elaboración de los diagramas de conexiones de los circuitos electrónicos. Con esta información se procedió a la construcción del prototipo en madera. Una vez armado, se realizaron las pruebas de funcionalidad, análisis de los resultados obtenidos y la calibración de la parte mecánica. De igual manera se realizó el estudio de mercado en la región, mediante encuestas, para determinar la aceptación del deshidratador entre los productores obteniendo resultados favorables.

Actualmente se está trabajando en un nuevo prototipo en acero inoxidable además de realizar el modelado matemático del efecto térmico que se produce en la estructura y el diseño básico de la aplicación móvil para, posteriormente realizar la prueba del funcionamiento del prototipo implementando loT, el análisis de los resultados obtenidos, la solución de posibles fallas mecánicas, electrónicas y de software para el análisis y comparación de los resultados obtenidos.

\section{Resultados y Discusión}

El prototipo diseñado es de tipo de deshidratador solar automatizado de colector y armario, realizado en madera que consta de un colector solar en el cual el aire se calienta y asciende hasta el armario donde se colocan los productos a deshidratar. El deshidratador está integrado de tres partes separadas para su fácil traslado: 1) Caja para la zona de secado, 2) Rampa o cama térmica y 3) Soporte inferior. El diseño preliminar se realizó en el programa SolidWorks para modelado mecánico en 2D y 3D. Cuenta con un sistema para el control del motor y el control automático se lleva a cabo, actualmente, mediante una tarjeta de adquisición de datos arduino que controla dos motorreductores con una capacidad de 21 kilogramos de fuerza. El deshidratador gira en torno al posicionamiento del sol a lo largo del día, lo que ayuda a que el proceso de deshidratación sea más rápido evitando la pérdida de producto y sin necesidad de personal para su vigilancia constante. 


\section{Figura 1}

Prototipo de deshidratador solar

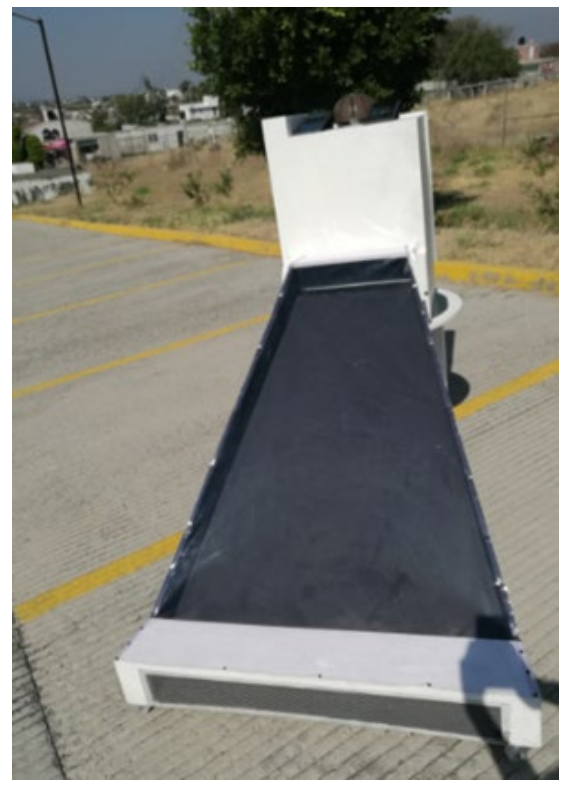

La caja de secado tiene 4 charolas de rejilla en su interior, cada una con su propia puerta. En la parte frontal de la caja de secado se encuentra una abertura para ensamblar la cama térmica, con un soporte acoplable para asegurar el ensamble y facilitar su movilidad. El escape se encuentra en la parte frontal izquierda, permitiendo circular el aire a la vez que retira la humedad de los jitomates. La parte inferior permite acoplar la caja con el soporte de la mesa mediante un espacio en donde se colocó un compartimiento para el mecanismo que genera el movimiento rotatorio del deshidratador y el sistema electrónico. La base de soporte inferior para la caja ensambla con la caja de alimentos. Esta pieza se mantendrá estática y está encargada de soportar las otras 2 piezas. La cama térmica, una vez montada, presenta un ángulo de inclinación de $35^{\circ}$ de acuerdo a lo recomendado en la página del Sistema de Información Geográfica Fotovoltaica de la Comisión Europea (2019), con la finalidad de captar la mayor cantidad de energía solar en el deshidratador, debido a que los paneles solares, al estar posicionados en un ángulo perpendicular a los rayos del sol, se vuelven más eficientes a la hora de generar energía eléctrica dando hasta un 15 o un 20 por ciento más de energía a la comparación de otros paneles solares con diferentes ángulos de inclinación. En la parte superior presenta una abertura para permitir el flujo de aire a la cama de productos.

Se emplearon motorreductores de tipo cónico-espiral o de velocidad de engranaje, para generar la fuerza necesaria para el giro del mecanismo. Los criterios de selección fueron: tienen mayor torque, menor velocidad y el voltaje y peso que se necesita es el indicado. La tarjeta de adquisición de datos es una tarjeta arduino por ser fácil de adquirir, económica y fácil de programar sin limitar sus funciones: control del funcionamiento del driver puente $h$, toma de datos de los sensores y envío de las señales correspondientes. El dispositivo para detectar los rayos solares, de forma cónica, cuenta con 10 aberturas para las fotorresistencias.

En las pruebas realizadas, con una temperatura ambiente de entre 21 y 29 grados centígrados, en un horario de 10 de la mañana a 5 de la tarde, los paneles solares arrojaron un voltaje pico de 23.8 volts $1 \mathrm{pm}$. El voltaje general fue de 21 volts, lo que ofrece una buena alimentación para mantener con carga las baterías. A partir de las $5 \mathrm{pm}$ el voltaje de los paneles solares disminuyó hasta 8.8 volts. La cama térmica alcanzó, entre 1 y 3 de la tarde una temperatura de 67 grados adecuada para el proceso de deshidratación. La capacidad del 
prototipo, con las dimensiones actuales, es de 7.2 kilos en rodajas de aproximadamente $1 \mathrm{~cm}$. de espesor que se deshidrataron completamente en 30 horas aprovechando los rayos solares al máximo por el movimiento giratorio del aparato.

Para la realización del estudio de mercado se realizó la aplicación de encuestas en el municipio de Atlatlahucan que, de acuerdo con México Red (2020), cuenta con 211 productores tan solo de jitomate y de los cuáles se encuestaron 176. Los resultados mostraron una aceptación para el deshidratador ya que todos ellos enfrentan la misma problemática de excedentes de producción. El 45\% $(\mathrm{n}=79)$ está interesado en adquirir el deshidratador para su uso. Respecto a la competencia solo existen dos empresas que elaboran productos similares, una de ella en el estado de Morelos y la otra en la ciudad de Puebla. Los productos que ofrecen son más sencillos, tienen dimensiones establecidas en dos y tres modelos y no cuentan con las características que definen nuestra ventaja competitiva que, en el prototipo actual, es su movimiento giratorio siguiendo el sol $\mathrm{y}$, una vez realizadas las mejoras, la nueva versión contará con controles que permiten programarlo a distancia y mantener registros detallados del proceso.

La utilidad por unidad es del $35 \%$. Con un nivel de ventas de 10 unidades de modelo de madera, al inicio del año 1 y al cierre de 15 , durante el primer año no se obtendrán utilidades debido a la inversión en maquinaria y equipo, sin embargo, con un promedio de ventas de 16 unidades a partir del segundo año se presentan utilidades.

\section{Discusión de resultados}

El deshidratado de frutas y legumbres toma más fuerza día con día. En el Estado de Sinaloa, la Fundación Produce Sinaloa, A.C., en conjunto con el gobierno del Estado, capacita a productores de mango y nopal en las técnicas de deshidratado y en la construcción de deshidratadores solares con el objetivo de preservar el producto durante muchos meses y consumirlo en periodos de escasez o fuera de temporada para evitar que se malogre y pierda su valor, además de obtener un producto refinado que asegure la calidad de alimentación familiar con un producto de alto valor nutritivo y libre de conservadores, "además aprovechar la energía solar y generar fuentes de empleo" (Pérez, s/f, pág. 8).

Por otra parte, López (2021), en Veracruz, propone un deshidratador solar cuya fuente de energía para el funcionamiento del sistema de control y comunicación parte de un sistema fotovoltaico en modalidad de autoabastecimiento, cuya fuente de calor es a través de resistencias eléctricas, mediante la implementación de un control de lazo cerrado para mantener la temperatura estable en la cámara de secado. Su diseño esta creado partir de la experiencia de usuario (UX) donde se implementa un display touch para el monitoreo y control del proceso de deshidratación para la selección de las opciones del tipo de alimento a deshidratar, la estimación del tiempo del proceso y la visualización de información en la nube, logrando mejorar la interacción entre el usuario y el producto y la reducción de tiempo de deshidratación a partir de flujo de aire caliente por convección forzada.

En España, la empresa navarra Trevijano, ofrece más de treinta productos deshidratados como: sopas, arroces, quinoa y cous cous; así como verduras y legumbres; ganando terreno con una oferta "desestacionalizada y de fácil manejo" (Redacción Interempresas, 2021), pero reconocen que el consumidor aún no está acostumbrado a este tipo de productos sanos, de buen sabor y fácil transporte si bien asocian las frutas deshidratadas, a celebraciones como la Navidad. Poco a poco, han ido logrando mayor presencia en los supermercados y más aceptación por parte de los consumidores. Señalan que este tipo de productos es "una buena manera de conservar los alimentos que salen del campo en su momento óptimo, por lo que se 
reduce el desperdicio alimentario, y permiten al consumidor utilizarlos en cualquier momento, ya que son fáciles de preparar y no necesitan estar refrigerados". Un $40 \%$ de las ventas de la empresa es para el mercado extranjero.

Jesús Torrealba (2021), especialista en nutrición señala múltiples beneficios de las frutas deshidratadas:

1. Al mantener intactos sus componentes potencian el sistema inmunológico protegiendo el organismo de infecciones.

2. Evitan la aparición de enfermedades crónica mediante el combaten de la acción de los radicales libres.

3. No contiene grasas regulando la presión arterial y mejorando la circulación sanguínea.

4. Contienen mucha fibra dietética lo que mejora las funciones digestivas.

5. Las frutas deshidratadas proveen vitamina $\mathrm{C}$, micronutriente esencial para la síntesis de colágeno; suministran calcio, que fortalece los huesos; así como magnesio, fósforo y zinc.

6. Aportan hasta $359 \mathrm{kcal}$ por cada ración de 100 gramos aumentando la energía física de rápida disponibilidad.

7. Ayudan a controlar el peso al aportar una menor cantidad de hidratos de carbono y grasas saturadas.

8. Las frutas deshidratadas tienen un poder antioxidante que favorece la salud de la piel protegiéndola de la aparición de arrugas prematuras causadas por el daño oxidativo a consecuencia de la exposición a los rayos solares.

Existen otros casos de nuevas empresas dedicadas a la producción de frutas y legumbres deshidratadas, sobre todo en América Latina, bajo diversos tipos de procesos, ya que la demanda crece día con día debido principalmente al ritmo de vida actual; por lo que el proyecto planteado tiene posibilidades de éxito y crecimiento.

\section{Conclusiones}

Como se puede apreciar la deshidratación de productos agrícolas se encuentra en pleno apogeo debido a sus múltiples ventajas y ya algunas empresas están ofreciendo este tipo de aparatos para su uso en el hogar, aunque estos aparatos funcionan con energía eléctrica.

El prototipo en cuestión presenta las ventajas de trabajar con energía solar, estar automatizado y ser programable. Se ofrece directamente a los productores agrícolas con la intención de dar un uso a lo que en este momento son desechos, lo que les permitirá disminuir sus costos de producción y diversificar sus productos y mercados ofreciendo al consumidor productos fuera de temporada, con las mismas propiedades alimenticias y a un menor costo. Adicionalmente se disminuye el problema de contaminación que generan actualmente este tipo de desechos.

Con las actuales dimensiones del prototipo este resulta ser muy funcional pero su gran ventaja es que puede escalarse al tamaño más adecuado de acuerdo con el uso que se le pretenda dar, desde más pequeño para uso doméstico o más grande con la finalidad de deshidratar más producto en el mismo tiempo mediante la adaptación de motorreductores con más fuerza para poder mover fácilmente la caja de secado. Si fuera necesario utilizar otro tipo de ruedas en la cama térmica para evitar la fricción al utilizar el aparato en diferentes tipos de suelo como terracería, ya que las pruebas se realizaron en superficies lisas. Las tres piezas del prototipo son armables y fáciles de ensamblar, dando la posibilidad de cambiar la cama térmica y la caja 
de secado en un tamaño más grande o pequeña dependiendo el área que se necesite y dando la oportunidad de una deshidratación más rápida incluso con espacio para más charolas.

Como se menciona al inicio de este trabajo, el proyecto contribuye al logro de las metas de los ODS de la Agenda 2030 por lo que adquiere una importancia desde el punto de vista social y económico. De igual manera impacta en el logro de los objetivos y cumplimiento de indicadores establecidos en el Programa Institucional de Desarrollo 2019-2024 (PND) del Tecnológico Nacional de México (TecNM) que establece en su Eje Estratégico 2 el Fortalecimiento de la investigación, el desarrollo tecnológico, la vinculación, el emprendimiento y la innovación para fortalecer la labor académica y contribuir a la generación del conocimiento, a través del desarrollo de productos, diseños, procesos y servicios que mejoran la calidad de vida de la sociedad; considerando entre los campos de acción aquellos en los que se pueden generar aportes sociales y económicos significativos a la agricultura, el medio ambiente, la electrónica y mecatrónica propiciando la vinculación con los sectores público, social y privado con el objetivo de contribuir al desarrollo social y sostenible así como al crecimiento económico de las regiones y, en general, del país (TecNM, s/f).

Por otra parte, y apoyando a lo establecido en el mismo PND, el TecNM ha incorporado en Modelo Educativo, temas como el emprendimiento, el cuidado del medio ambiente, la biodiversidad y el entorno sostenible promoviendo aportaciones que contribuyan al manejo de desechos mediante la prevención, reducción, reciclaje y reutilización, así como la utilización de energías renovables; aspectos todos contemplados en este proyecto. Al mismo tiempo, el proyecto favorece el trabajo multidisciplinario de docentes y estudiantes desarrollando en ellos nuevas habilidades y competencias.

\section{Reconocimientos}

Este trabajo ha sido financiado por el Tecnológico Nacional de México (TecNM) dentro de la Convocatoria 2021-2 Proyectos de Investigación Científica, para los Institutos Tecnológicos Federales, Descentralizados y Centros que tiene como finalidad: apoyar e impulsar la investigación científica, el desarrollo tecnológico e innovación, permitiendo a docentes y estudiantes el desarrollo y puesta en práctica de sus ideas. Las autoras desean expresar su agradecimiento al resto del equipo que participa en el trabajo y a las autoridades del Campus Cuautla del TecNM.

\section{Referencias}

2000 Agro. (31 de julio de 2018). Logra Morelos cifra histórica en producción agrícola. Recuperado el febrero de 2021, de http://www.2000agro.com.mx/politicaagropecuaria/logra-morelos-cifra-historica-enproduccion-agricola/

Cajamarca, D., Mendoza, J., \& Baño, D. (junio de 2019). La Calidad una metodología innovadora y sostenible en la producción de frutas deshidratadas. Obtenido de Revista Observatorio de la Economía Latinoamericana: https://www.eumed.net/rev/oel/2019/06/produccion-frutas-deshidratadas.html

Carreño, G. (1 de diciembre de 2020). El sector agropecuario se mantiene creciendo en Morelos. El Sol de Cuernavaca, pág. 2.

Cocimaniacos. (19 de junio de 2017). Temperaturas y tiempos de deshidratación de alimentos en un deshidratador. Recuperado el julio de 2021, de https://cocimaniacos.com/temperaturas-y-tiempos-dedeshidratacion-de-alimentos-en-un-deshidratador/

Comercio justo. (5 de agosto de 2019). El consumo y la producción sostenibles fomenta el progreso de todos los Objetivos de Desarrollo Sostenible. (Coordinadora Estatal de Comercio Justo) Obtenido de 
http://comerciojusto.org/el-consumo-y-la-produccion-sostenibles-fomenta-el-progreso-de-todos-losobjetivos-de-desarrollo-sostenible

Comisión Europea. (15 de octubre de 2019). Sistema de Información Fotovoltaica. Obtenido de Comisión Europea: https://re.jrc.ec.europa.eu/pvg_tools/en/tools.html

El Sol de Cuernavaca. (22 de julio de 2018). Logra Morelos cifra histórica en producción agrícola. El Sol de Cuernavaca. Obtenido de https://www.elsoldecuernavaca.com.mx/local/logra-morelos-cifra-historicaen-produccion-agricola-1858820.html

Fede Fruta. (2018). Consumo Mundial de frutas deshidratadas sería de 4 millones de toneladas en 2020. Obtenido de Fede fruta: https://fedefruta.cl/consumo-mundial-de-frutasdeshidratadas-

INEGI. (2019). Agricultura, ganadería y pesca. Obtenido de INEGI: https://www.inegi.org.mx/temas/agricultura/

INTA. (2018). La Deshidratación. Obtenido de Cursos de autoaprendizaje: http://procadisaplicativos.inta.gob.ar/cursosautoaprendizaje/deshidratacion/17_p2.html

La redacción. (22 de julio de 2018). Logra Morelos cifra histórica en producción agrícola. El Sol de Cuautla. Obtenido de https://www.elsoldecuautla.com.mx/local/logra-morelos-cifra-historica-en-produccionagricola-1858820.html\#!

López, D. (10 de julio de 2021). Deshidratador de alimentos híbrido automatizado con perspectiva de diseño de usuario. Obtenido de Congreso Nacional de secado y cocción solar de alimentos: https://consycsa.com/deshidratador-de-alimentos-hibrido-automatizado-con-perspectiva-de-diseno-deusuario

Más de Morelos. (4 de diciembre de 2017). Morelos, primer lugar nacional en la producción de estos 7 cultivos. Obtenido de + de Morelos: https://masdemorelos.masdemx.com/2017/12/morelos-primerlugar-nacional-produccion-cultivos-arroz-nopal-nochebuena/

México Red. (2020). Obtenido de http://mexicored.com.mx/send_message.php?type=tag\&id=34393

Montesino, L. (2018). Frutas deshidratadas: Qué es y como se fabrica. Obtenido de Comefruta: https://comefruta.es/fruta-deshidratada-que-es

ODS. (s/f). Objetivo 12: Garantizar modalidades de consumo y producción sostenibles. Obtenido de ONU: https://www.un.org/sustainabledevelopment/es/sustainable-consumption-production/

ONU. (2019). Informe de los Objetivos del desarrollo Sostenible 2019. Obtenido de Organización de las Naciones Unidas: https:/unstats.un.org/sdgs/report/2019/The-Sustainable-Development-Goals-Report2019_Spanish.pdf

ONU. (s/f). Agenda 2030. Obtenido de Organización de las Naciones Unidas: http://www.onu.org.mx/agenda2030/

Orellana, J. (noviembre de 2017). Automatización y control de un deshidratador solar. Valparaiso, Chile: Universidad Técnica Federico Santa María. Obtenido de https://repositorio.usm.cl/bitstream/handle/11673/47435/3560900251250UTFSM.pdf?sequence=1

Pacto por la comida. (s/f). Pacto por la comida. Obtenido de https://www.pactoporlacomida.org/

Pérez, L. M. (s/f). Técnicas para el Deshidratado de mango. (A. Fundación Produce Sinaloa, Ed.) Memoria de capacitación.

PROECUADOR. (marzo de 2018). Mango, piña, uvilla y banana deshidratada en el mercado de Estados Unidos. Recuperado el mayo de 2021, de https://www.proecuador.gob.ec/mango-pina-uvilla-ybananadeshidratada-en-el-mercado-de-los-estados-unidos-2018/. 
Redacción Interempresas. (1 de septiembre de 2021). Las frutas y verduras deshidratadas, una oferta en crecimiento. Obtenido de Interempresas: https://www.interempresas.net/Alimentaria/Articulos/358705Las-frutas-y-verduras-deshidratadas-una-oferta-en-crecimiento.html

Rivera López, O. (2 de enero de 2018). ¿Sabes cuantas toneladas de comida tira México a la basura? El Universal. Recuperado el diciembre de 2018, de https://www.eluniversal.com.mx/nacion/sociedad/mexico-desperdicia-20-millones-de-toneladas-decomida

TecNM. (s/f). Programa de Desarrollo Institucional PDI 2019-2024. CDMX: Tecnológico Nacional de México.

Torrealba, J. (6 de agosto de 2021). Frutas deshidratadas: propiedades y beneficios para la salud. Obtenido de Nutrición sin más: ttps://nutricionsinmas.com/frutas-deshidratadas/ 